\title{
Liquen plano, estudio retrospectivo en un hospital pediátrico
}

\section{Lichen planus, retrospective study in a pediatric hospital}

María José Pertuz Suárez ${ }^{1}$, María Marta Buján ${ }^{2}$, María del Valle Centeno ${ }^{3}$ y Andrea Bettina Cervini ${ }^{4}$

\section{RESUMEN}

Introducción: El liquen plano (LP) es una enfermedad inflamatoria crónica, de etiología desconocida, que puede afectar la piel, los anexos y las mucosas.

Objetivo: Describir las características epidemiológicas y clínicas y el tratamiento realizado en los pacientes con diagnóstico clínico e histopatológico de LP, valorados en el Servicio de Dermatología del Hospital de Pediatría "Prof. Dr. Juan P. Garrahan" en los últimos 33 años. Diseño: Estudio descriptivo, retrospectivo y transversal.

Materiales y métodos: Se revisaron las historias clínicas de los pacientes con diagnóstico histopatológico de LP atendidos en el Hospital desde agosto de 1987 hasta febrero de 2020 y se analizaron las variables epidemiológicas, clínicas y de tratamiento.

Resultados: Se incluyeron datos de 26 pacientes, 18 de los cuales eran varones (69,3\%). La media de edad en el momento del diagnóstico fue de 9,6 años. Presentaron la variedad de LP clásico 24 pacien- tes $(93 \%)$. El compromiso ungueal y de la mucosa bucal se observó en 2 pacientes (7,7\%). Ninguno tuvo asociación a fármacos ni a infecciones. En cuanto al tratamiento recibido, 4 pacientes $(15,4 \%)$ tuvieron pérdida del seguimiento; 13 (50\%) realizaron tratamiento tópico; $6(23 \%)$, tratamiento sistémico y $3(11,5 \%)$, tratamiento combinado. El promedio de seguimiento fue de 6 meses y se obtuvo una respuesta favorable en el $68 \%$ de los pacientes.

Conclusiones: El LP es una patología de baja frecuencia en la infancia. No se encontró asociación a medicamentos ni a enfermedades infecciosas como se describe en la población adulta. Su curso es crónico y, en los pacientes que tuvieron un adecuado seguimiento, se obtuvo una buena respuesta al tratamiento indicado.

Palabras clave: liquen plano, niños, revisión.

Dermatol. Argent. 2021, 27 (2): 64-68

\section{ABSTRACT}

Introduction: Lichen planus (LP) is a chronic, inflammatory disease of unknown etiology, which can affect skin, adnexal tissues and mucosa. Objective: to describe the clinical, epidemiological characteristics and the treatment received by patients with clinical and histopathological diagnosis of $L P$, seen at the Dermatology Service of Pediatric Hospital "Prof. Dr. Juan P. Garrahan" in the last 33 years.

Design: Descriptive, retrospective and cross-sectional study.

Materials y methods: We reviewed the clinical history of patients with histopathological diagnosis of $L P$ that were treated at our hospital from August 1987 to February 2020 and analysed the epidemiological, clinical and treatment variables.

Results: Data of 26 patients were included in this study 18 of which are males (69.3\%). The mean age of diagnosis was 9.6 years. 24 patients (93\%) presented the classical type of LP. Ungual and mucosal compromise were observed in 2 patients (7.7\%) and none had associations with infections or drugs. Regarding treatment, 4 patients (15.4\%) did not continue follow-ups, 13 patients (50\%) had topical treatment, 6 (23\%) had systemic treatment and 3 patients (11.5\%) received combined treatment. The average follow-up time was 6 months and $68 \%$ of patients had favorable outcome.

Conclusions: LP is an uncommon pathology in children. During this study we did not find any etiologic relations with use of drugs or infectious diseases as is described in de adult population. Its course is chronic and in the patients that had a proper follow-up a good response to the indicated treatment was obtained.

Key words: lichen planus, children, revision.

Dermatol. Argent. 2021, 27 (2): 64-68 
${ }^{1}$ Médica Becaria de Tercer Año, Servicio de Dermatología

2 Médica de Planta, Servicio de Dermatología

${ }^{3}$ Médica de Planta, Servicio de Patología

4 Jefa, Servicio de Dermatología

Hospital Nacional de Pediatría Prof. Dr. Juan P. Garrahan, Ciudad Autónoma de Buenos Aires, Argentina
Contacto de la autora: María José Pertuz Suárez

E-mail: majo_pertuz@hotmail.com

Fecha de trabajo recibido: 22/5/2021

Fecha de trabajo aceptado: 28/6/2021

Conflicto de interés: las autoras declaran que no existe conflicto de interés.

\section{INTRODUCCIÓN}

El liquen plano (LP) es una enfermedad inflamatoria, crónica, autolimitada, de etiología desconocida, que puede comprometer la piel, las mucosas, los pelos y las uñas. Tiene una prevalencia menor del $1 \%$ y afecta con mayor frecuencia a los adultos que a los nińos ${ }^{1}$. Clínicamente, las lesiones se presentan como pápulas o placas planas, violáceas, poligonales, brillantes y pruriginosas, ubicadas de preferencia en los miembros ${ }^{2}$. Se ha asociado esta enfermedad a infecciones, entre ellas, la hepatitis $\mathrm{C}$ y la sífilis, más observadas en la población adulta. El tratamiento se basa en los corticosteroides de alta potencia en los casos localizados y en la fototerapia en los generalizados. También se describió el uso de los corticosteroides sistémicos, la acitretina o los inmunosupresores orales para casos más severos y refractarios al tratamiento ${ }^{3}$. El LP puede resolverse de manera espontánea al cabo de 1-2 años ${ }^{3}$. Sin embargo, las recurrencias son comunes.

El objetivo de este trabajo fue determinar las características epidemiológicas y clínicas, así como el tratamiento realizado en los pacientes con diagnóstico clínico e histopatológico de LP, evaluados en el Servicio de Dermatología del Hospital de Pediatría "Prof. Dr. Juan P. Garrahan" en los últimos 33 años.

\section{MATERIALES Y MÉTODOS}

Se realizó un estudio descriptivo, retrospectivo, observacional y de corte transversal, en el que se analizaron las historias clínicas de los pacientes con diagnóstico de LP confirmado por histología, atendidos en el Servicio de Dermatología desde agosto de 1987 hasta febrero de 2020. Se evaluaron las siguientes variables: sexo; edad en la primera consulta; antecedentes personales de relevancia; tiempo de evolución hasta la primera consulta; características clínicas; variantes morfológicas y extensión de las lesiones; compromiso de los anexos y las mucosas; presencia de síntomas; antecedentes de ingestión de fármacos, de asociación con infecciones o de otras enfermedades; tratamiento realizado; seguimiento y evolución.

\section{RESULTADOS}

Durante el período estudiado, se evaluaron 26 pacientes con diagnóstico clínico e histopatológico de LP. De estos, 18 pacientes $(69,3 \%)$ fueron varones y $8(30,7 \%)$, mujeres. La edad promedio en el momento de la primera consulta fue de 9,6 ańos, con un rango entre 3 y 17 años (Gráfico 1). Como antecedentes personales de relevancia, 3 pacientes $(11,5 \%)$ tenían enfermedades neurológicas asociadas (síndrome convulsivo, trastorno generalizado del desarrollo y retraso madurativo), 1 paciente $(3,8 \%)$ vitiligo y 1 paciente $(3,8 \%)$ dermatitis atópica. Si bien el tiempo de evolución promedio de la enfermedad en el momento de la primera consulta fue de 23 meses (3 días4 años), el $50 \%$ de los pacientes consultaron dentro de los 3 primeros meses de aparecidas las lesiones (Gráfico 2). La presentación clínica observada con mayor asiduidad fue la del LP clásico en 24 pacientes $(92,3 \%)$, mientras que solo 2 pacientes $(7,7 \%)$ presentaron la variante hipertrófica. En 23 pacientes $(88,5 \%)$ el compromiso fue generalizado y solo en 3 pacientes $(11,5 \%)$, localizado (planta del pie, muñeca y tobillo). Junto con la afectación cutánea, se observó compromiso ungueal y de la mucosa bucal en 2 pacientes $(7,7 \%)$, respectivamente.

El prurito fue referido por la mayoría de los pacientes $(84,6 \%, 22$ pacientes). No se observaron en

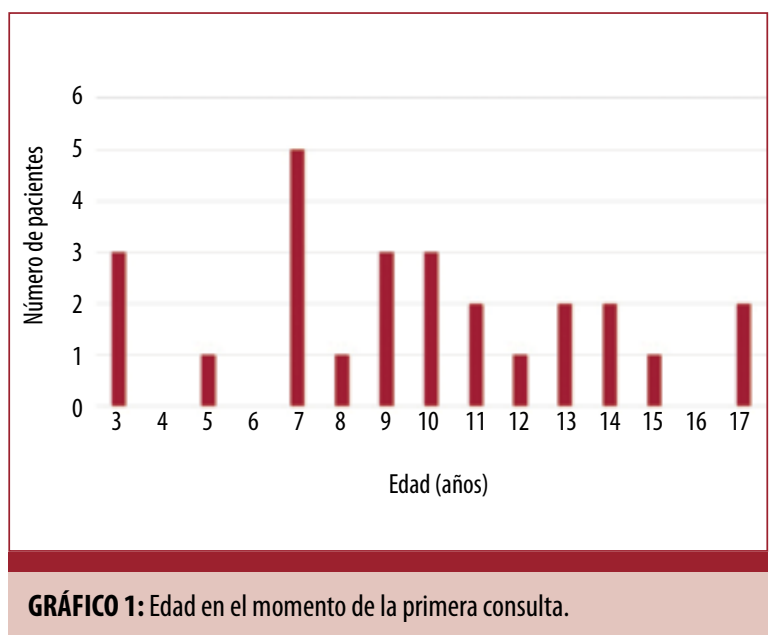


nuestra cohorte asociaciones a fármacos, infecciones ni enfermedades hepáticas.

En cuanto al tratamiento instaurado, 4 pacientes $(15,4 \%)$ tuvieron pérdida del seguimiento, por lo que no pudo indicarse tratamiento. De los 22 pacientes restantes, 13 (59\%) realizaron solo tratamiento tópico; 6 (27\%), tratamiento sistémico únicamente y 3 (14\%), tratamiento combinado (Gráfico 3).

De los 13 pacientes que realizaron solo tratamiento tópico, todos recibieron humectación, $10(76,9 \%)$ recibieron, además, corticosteroides de mediana y alta potencia, y $1(7,7 \%)$, inhibidores de la calcineurina (tacrolimus). Del total de pacientes que recibieron tratamiento sistémico $(n=6), 4$ de ellos $(66,6 \%)$ realizaron fototerapia UVB-NB (en promedio, 23 sesiones), 1 paciente $(16,7 \%)$ recibió griseofulvina y otro $(16,7 \%)$ realizó fototerapia (40 sesiones) junto con el tratamiento con corticosteroide sistémico (metilprednisona en dosis de $1 \mathrm{mg} / \mathrm{kg} /$ día durante una semana). La totalidad de los pacientes que recibieron el tratamiento combinado
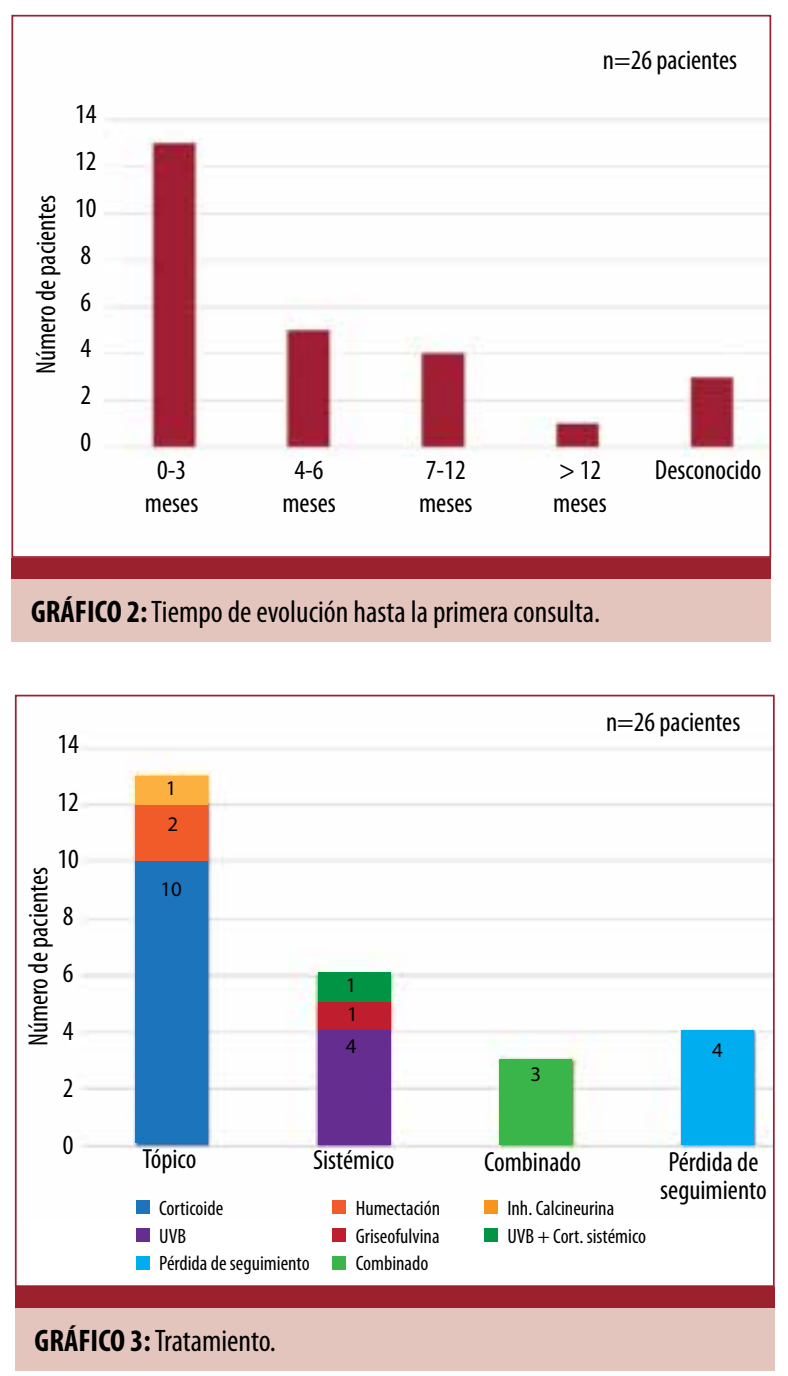

$(n=3)$ realizaron fototerapia UVB-NB y tomaron un corticosteroide tópico de alta potencia (clobetasol).

El tratamiento indicado para el compromiso ungueal fue el ácido retinoico al $0,025 \%$, junto con triamcinolona al $0,1 \%$ en crema y, para el compromiso de la mucosa bucal, triamcinolona al $0,1 \%$ en orabase.

Debido a la presencia de prurito, 16 pacientes $(61,5 \%)$ fueron medicados con antihistamínicos.

La respuesta a los tratamientos indicados se pudo determinar en 22 pacientes, ya que 4 no tuvieron un seguimiento posterior al diagnóstico. El promedio del seguimiento fue de 6 meses ( 1 a 20 meses). Del total de pacientes (22), 15 (68\%) presentaron una evolución favorable y, de estos, 7 (46,6\%) tuvieron una resolución completa de las lesiones y $8(53,4 \%)$, una respuesta parcial. Siete pacientes (32\%) sufrieron un empeoramiento de las lesiones.

\section{COMENTARIO}

El LP es una enfermedad inflamatoria, crónica y autolimitada, que puede afectar la piel, sus anexos y la mucosa genital u oral. Su etiopatogenia permanece poco clara; ahora se lo considera un trastorno inmunomediado de naturaleza multifactorial ${ }^{4}$. Es una enfermedad poco frecuente en la infancia, ya que se presenta en el $1 \%$ de la población general y, de estos, el $1-2 \%$ corresponde a pacientes en edad pediátrica, lo cual coincide con nuestra experiencia, ya que en 33 ańos solo hemos atendido a 26 pacientes con esta afección ${ }^{1}$. En nuestra casuística, encontramos una predilección por el sexo masculino, similar a lo comunicado por Sharma et ál. y Nanda et ál. en sus series de $50 \mathrm{y}$ 23 pacientes, respectivamente ${ }^{5,6}$.

El LP clásico se manifiesta como pápulas y placas aplanadas, poligonales y violáceas, localizadas de preferencia en las superficies flexoras del tronco, los miembros (Foto 1) y los genitales ${ }^{2,7,8}$. Menos a menudo, se presenta la variedad hipertrófica, que predomina en los miembros inferiores y tiene una apariencia verrugosa (Foto 2$)^{2}$. Otras variantes, mucho menos frecuentes en pediatría, son: actínico, pigmentado, anular, lineal, ampollar y pilaris. Además, existen las variantes de LP oral y ungueal, que pueden aparecer hasta en un 10\% de los casos como afectación exclusiva de estas localizaciones. En la superficie de las lesiones, se pueden observar líneas blanquecinas conocidas como estrías de Wickham².

Esta dermatosis se caracteriza, asimismo, por presentar el fenómeno de Koebner (véase Foto 1$)^{9,10}$. En cuanto a la variedad morfológica de presentación, en nuestra casuística predominó la variedad clásica, lo que coincide con los datos publicados ${ }^{5,6}$. Con respec- 
to a la distribución de las lesiones, al contrario de lo publicado, en que la forma localizada es la más frecuente, nuestros pacientes presentaron más a menudo la forma generalizada ${ }^{5,6}$. El compromiso ungueal y de la mucosa bucal en nuestra casuística fue de baja frecuencia, al igual que lo informado en la bibliografía revisada (Fotos 3 y 4$)^{5,8-11}$.

Las lesiones son intensamente pruriginosas. Nosotros encontramos que el $85 \%$ de los pacientes referían prurito, cifra similar a lo señalado por Tilly et ál. y por Pandhi et ál., quienes hallaron este síntoma hasta en el $80 \%$ y el $94 \%$ de los niños, respectivamente ${ }^{7,12}$.

La histopatología del LP en el nińo no difiere de la del adulto. Se observa una epidermis con hiperqueratosis ortoqueratósica, hipergranulosis en cuña, acantosis irregular con aspecto de "dientes de sierra" y vacuolización de la capa basal. En la unión dermoepidérmica, se pueden observar los cuerpos de Civatte, los cuales representan queratinocitos apoptóticos. En la dermis papilar,

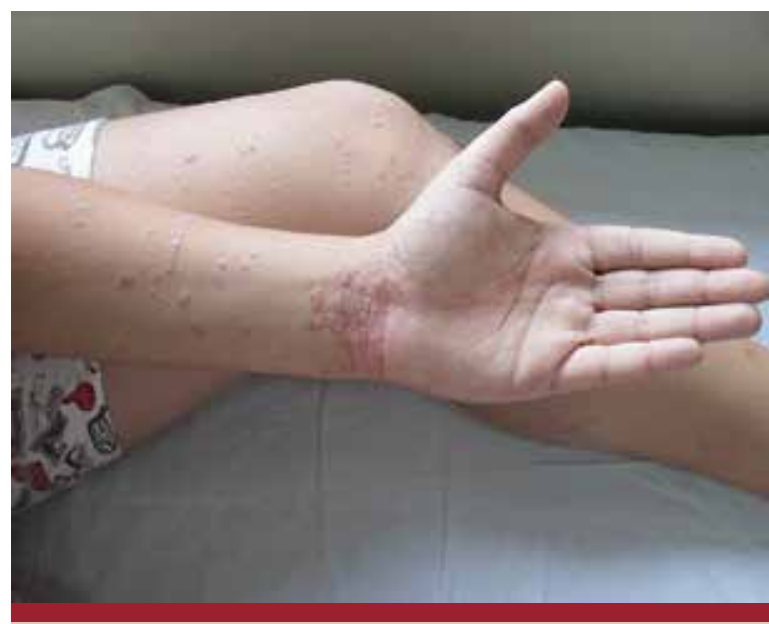

FOTO 1: Liquen plano clásico. Pápulas y placas aplanadas eritematovioláceas, brillantes, con el fenómeno de Koebner.

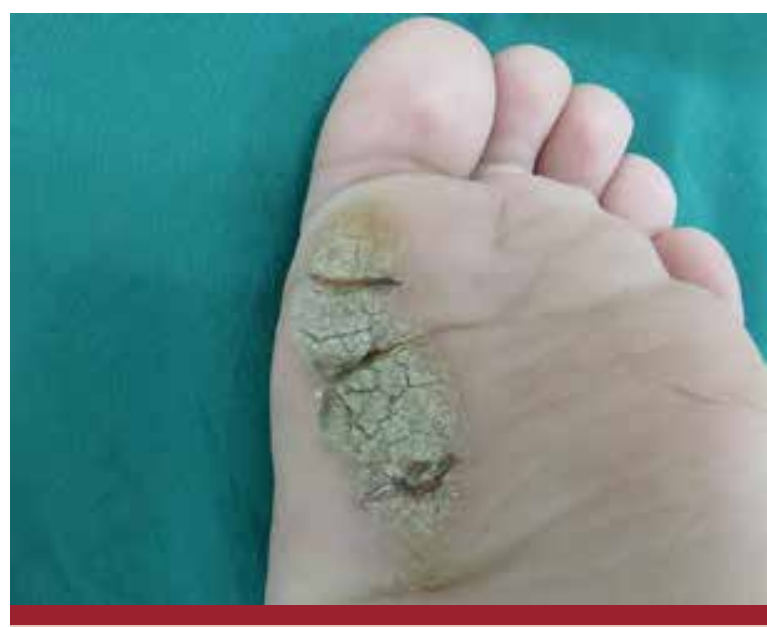

FOTO 2: Liquen plano hipertrófico. Placa hipertrófica, verrugosa y con fisuras. acompaña a estos cambios un denso infiltrado inflamatorio de linfocitos dispuestos en banda (Foto 5) $)^{1,29,11,13}$.

Se describieron con mayor frecuencia asociaciones del LP a enfermedad hepática crónica, infecciones

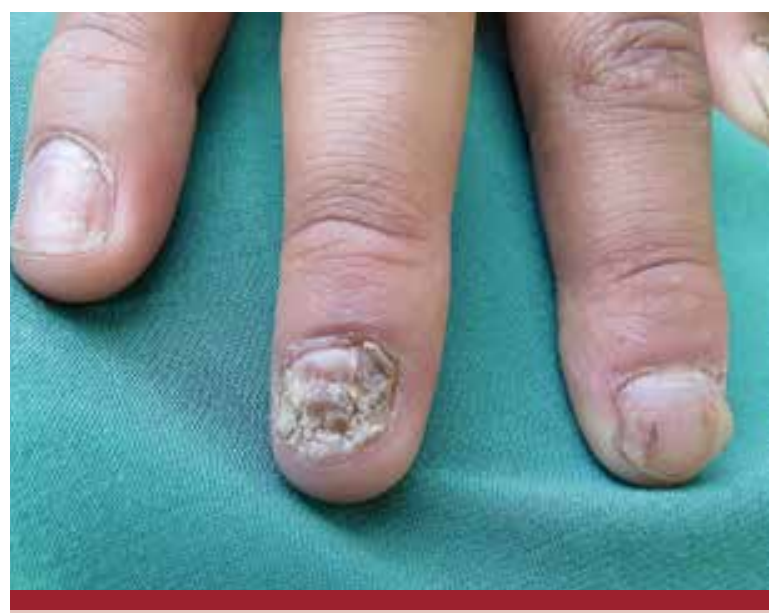

F0T0 3: Liquen plano ungueal. Hiperqueratosis, onicorrexis, fragmentación de la lámina ungueal y onicosquisis.

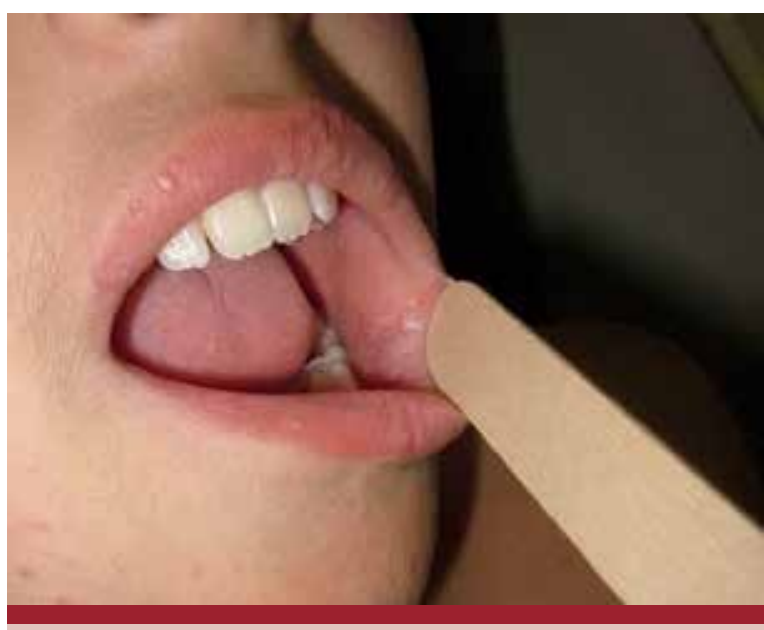

FOTO 4: Liquen plano mucoso. Pápulas blanquecinas aplanadas de disposición reticular.

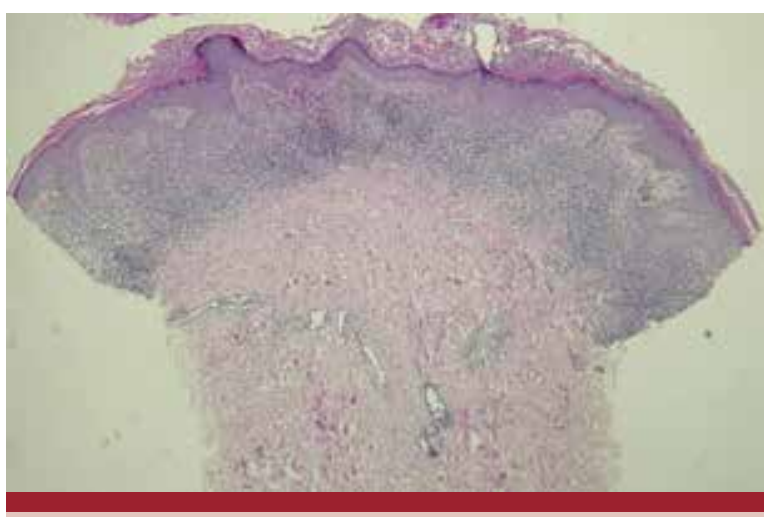

F0T0 5: Histopatología. Hiperqueratosis, focos de hipergranulosis e infiltrado liquenoide en banda que borra la interfaz (HyE, 40X). 
virales (hepatitis B y C, EBV, CMV), sífilis, fármacos (antipalúdicos, betabloqueantes, estatinas, diuréticos tiazídicos) y también secundario a la vacunación contra la hepatitis $B^{6,7,10,14}$. En nuestro estudio, al igual que en el de Nanda et ál. y en el de Pandhi et ál., no encontramos tales asociaciones ${ }^{6,12}$.

El tratamiento de esta entidad varía según su extensión. En los casos localizados, los corticosteroides tópicos son los de elección ${ }^{6-8,10}$. En cambio, en los casos de distribución generalizada, lo es la fototerapia $^{7,10,12}$. Se han planteado otras opciones terapéuticas de segunda y tercera línea, como los corticosteroides sistémicos, la acitretina, la griseofulvina, la hidroxicloroquina y la azatioprina, reservados para los pacientes

\section{BIBLIOGRAFÍA}

1. Carriquirri F, Villoldo V, Allevato $\mathrm{M}$, Cabrera H. Liquen plano lineal. Tratamiento con tacrolimus tópico. Act Terap Dermatol 2006;29:395-399.

2. Gru AA, Salavaggione AL. Lichenoid and interface dermatoses. Semin Diag Pathol 2017;34:237-249.

3. Cribier B, Frances C, Chosidow O. Treatment of lichen planus. An evidence-based medicine analysis of efficacy. Arch Dermatol 1998;134:1521-1530.

4. European Dermatology Forum. EDF S1 Guidelines on the management of lichen planus. Disponible en: https://www.edf. one/dam/jcr:263c4016-0be7-4b2c-bddd-1 c65e9633965/EDF_ Guidelines_Lichen_planus_final.pdf [consulta: abril 2021].

5. Sharma R, Maheshwari V. Childhood lichen planus: a report of fifty cases. Pediatr Dermatol 1999;16:345-348.

6. Nanda A, Al-Ajmi H, Al-Sabah H, Al-Hasawi F, et ál. Childhood lichen planus: a report of 23 cases. Pediatr Dermatol 2001;8:1-4.

7. Tilly J, Drolet B, Esterly N. Lichenoid eruptions in children. J Am Acad Dermatol 2004;51:606-624. con mala respuesta a los tratamientos iniciales, o la dapsona, indicada para los pacientes con LP recurrente y crónico ${ }^{3,4,7,10,11}$. También se observó la resolución espontánea de las lesiones, no hallada en ninguno de nuestros pacientes.

El LP es una enfermedad inflamatoria de baja frecuencia en la infancia que compromete la piel, las mucosas o los anexos, de curso crónico, con características clínicas típicas y que presenta múltiples opciones terapéuticas según su extensión. Si bien en nuestro estudio se obtuvo una buena respuesta en los pacientes que tuvieron un seguimiento adecuado, debido a su diseño no fue posible sacar conclusiones en relación con la efectividad de los distintos tratamientos instaurados.

8. Ramirez L, Franco AC, López NE, Proy Trujillo H, et ál. Liquen plano: perfil clínico y características demográficas de 129 casos en el Centro Dermatológico de Yucatán. Dermatología CMQ 2016;14:185-190.

9. Hamade $\mathrm{Y}$, Arita $\mathrm{K}$, Toyonaga $\mathrm{E}$, Inokuma $\mathrm{D}$, et ál. Lichen planus in childhood showing various cutaneous features. Acta Derm Venereol 2012;92:386-387.

10. Payette $\mathrm{M}$, Weston $\mathrm{G}$, Humphrey $\mathrm{S}, \mathrm{Yu} \mathrm{J}$, et ál. Lichen planus and other lichenoid dermatoses: Kids are not just little people. Clin Dermatol 2015;33:631-643.

11. Dahbar M, García Díaz R, Laffargue J, Laterza A, et ál. Tratamiento de liquen plano con griseofulvina. Arch Argent Dermatol 2002;52:23-26.

12. Pandhi $D$, Singal $A$, Bhattacharya $S$. Lichen planus in childhood: a series of 316 patients. Pediatr Dermatol 2014;31:59-67.

13. Wackernagel $A$, Legat $F$, Hofer $A$, Quehenberger $F$, et ál. Psoralen plus UVA vs. UVB-311 $\mathrm{nm}$ for the treatment of lichen planus. Photodermatol Photoimmunol Photomed 2007;23:15-19.

14. Kanwar A, De D. Lichen planus in childhood: report of 100 cases. Clin Exp Dermatol 2010;35:257-262.

\section{PERLAS}

Federico Pastore

Médico Dermatólogo y Legista. Médico de Planta de Dermatología, Hospital Dr. Enrique Tornú.

Jefe del Servicio de Dermatología, Instituto César Milstein

\section{EFICACIA DE UN SEGUNDO INHIBIDOR DELA} INTERLEUCINA-17 EN PACIENTES CON PSORIASIS

Entre los numerosos tratamientos biológicos para la psoriasis, los inhibidores de la interleucina-17 (IL-17) son seguros y altamente efectivos. A pesar de ello, en ocasiones es necesario cambiar el tratamiento. Se evaluó la eficacia de un inhibidor de la IL-17 en pacientes que habían presentado falla primaria 0 secundaria al tratamiento con otro inhibidor de la IL-17. Los ensayos clínicos analizados no mostraron diferencias de eficacia entre los pacientes que nunca habían recibido tratamiento y aquellos ya medicados con otro inhibidor de la IL-17. En la práctica clínica, la eficacia es levemente inferior en los ya tratados con fármacos biológicos.

En las publicaciones estudiadas, el porcentaje de reducción del PASI 75, 90 y 100 fue de 74,6, 69,4 y 46,4 respectivamente, a las semanas 9,12 y 16 . En conclusión, un tratamiento previo con un inhibidor de la IL-17 no parece afectar la eficacia de otro inhibidor de la IL-17 en los pacientes con psoriasis.

Loft N, Halling A, Egeberg A, Skov L. Efficacy of a second interleukin 17 inhibitor in patients with psoriasis: A systematic review and meta-analysis. J Am Acad Dermatol 2021;84:130-138.

\section{TRATAMIENTO DE LA NECRÓLISIS EPIDÉRMICA} TÓXICA CON INMUNOMODULADORES SISTÉMICOS

El síndrome de Stevens-Johnson (SSJ) y la necrólisis epidérmica tóxica (NET) son reacciones cutaneomucosas severas caracterizadas por necrosis epidérmica y elevada mortalidad.

La fisiopatogenia es inmunitaria, por lo que para su tratamiento se han utilizado diferentes inmunomoduladores como corticosteroides, inmuno- globulinas, ciclosporina, talidomida, etanercept, infliximab, plasmaféresis, solos o combinados, con una eficacia variable.

La talidomida evidenció una mortalidad significativamente mayor en comparación con las medidas de sostén.

La combinación de corticosteroides e inmunoglobulina intravenosa reduce el riesgo de mortalidad en los pacientes. Otros tratamientos, como la ciclosporina sola o combinada con inmunoglobulinas, 0 estas últimas combinadas con plasmaféresis y etanercept, serían efectivos en los pacientes con NET o con el síndrome de superposición SSJ/NET.

Tsai TY, Huang IH, Chao YC, Li H, et ál. Treating toxic epidermal necrolysis with systemic immunomodulating therapies: A systematic review and network metaanalysis. J Am Acad Dermatol. 2021;84:390-397. 